\title{
COMPETITIVE GRAIN GROWTH IN DIRECTIONAL SOLIDIFICATION OF A NICKEL-BASE SUPERALLOY
}

\author{
Y.Z. Zhou, N.R. Green \\ School of Engineering, Metallurgy and Materials, University of Birmingham, Edgbaston, Birmingham B15 2TT, UK
}

Keywords: directional solidification; seeding casting; dendrite growth; grain growth

\begin{abstract}
The structure evolution of bi-crystal samples during directional solidification was explored. It was found that in the case of diverging dendrites the better aligned grains overgrew the misaligned grains by means of the dendrite development from the better aligned grains. However, in the case of converging dendrites the result differs in two ways from the prediction of the generally accepted model for competitive grain growth. First, the misaligned dendrites are able to overgrow the better aligned dendrites. Second, the misaligned grains are able to overgrow the better aligned grain by blocking the dendrites of the better aligned grains at the grain boundary. The results can explain the potential sources of misorientation defects in seeded single crystal components and can be used to optimize process design to eliminate the stray grain defects in seeded single crystal casting.
\end{abstract}

\section{Introduction}

Turbine blades are routinely cast from nickel-base superalloys. To improve creep-rupture, fatigue, oxidation and coating properties, the modern blades are often cast in single crystal (SC) form, which results in superior turbine engine performance and durability $[1,2]$. In certain circumstances pertaining to mechanical response, it is necessary to obtain an $<001>$ orientation within a component that is misaligned with respect to the withdrawal direction and temperature gradient, the normal determinants of preferred crystal growth direction [3]. This is achieved by means of a seeding technique.

In commercial production, the prefabricated seed with desired orientation is partially melted back in the mould during the thermal soaking period prior to casting. Subsequently, the molten alloy is introduced through the mould cavity across the seed "melt-back" interface and the mould is then withdrawn from the furnace. It was reported $[4,5]$ that upon initiation of withdrawal, copious nucleation occurred, of crystals with random orientation distributed uniformly around the perimeter of the seed at the meltback interface. In the following growth process [4, 5], stray grains survived and expanded transversely on one side of the seed. Stray grains grew on the side of the seed where the primary dendrites of the seed diverged from the mould wall. The stray grains were overgrown by the seed crystal (Figure 1) where the dendrites converged on the mould wall. A general model for competitive grain growth, the Walton and Chalmers model [6], can explain why the stray grains survive and expand transversely on the diverging side, but it cannot explain why stray grains better aligned to the withdrawal direction and thermal gradient were overgrown by the misaligned seed on the converging side.

The Walton and Chalmers model suggests that the process of grain overgrowth is based on the difference in undercooling of favourably and unfavourably oriented dendrites with respect to the thermal gradient, as shown in Figure 2 [7]. Grains $A_{1}$ and $A_{2}$ are favorably oriented, while grain $\mathrm{B}$ is unfavorably oriented and the $<001>$ direction has a misorientation $(\theta)$ with respect to the heat flow direction. To keep up with the better aligned neighbors, grain $\mathrm{B}$ grows at a greater undercooling. In the case of diverging dendrites, the two grains on the right of Figure 2, development of new dendrites from grain $A_{2}$ can lead to overgrowth of grain $B$ and the grain boundary $(\mathrm{GB})$ is thus inclined. In the case of converging dendrites, the two grains on the left of Figure 2, the dendrite tips in grain $\mathrm{B}$ impinge upon the side of grain $\mathrm{A}_{1}$ at the $\mathrm{GB}$ and are stopped. Since grain $\mathrm{A}_{1}$ does not develop new dendrites at the $\mathrm{GB}$ and the dendrites in grain $\mathrm{B}$ cannot overgrow the dendrites in grain $A_{1}$, the GB lies parallel to the dendrites in grain $\mathrm{A}_{1}$.

In our work [8], structure evolution of bi-crystal (BC) samples during the DS process was explored in an attempt to understand the mechanism of competitive grain growth. It was found that in the case of diverging dendrites the best aligned grain overgrew the misaligned grain. This result was in accordance to the Walton and Chalmers model. However, in the case of converging dendrites the result differed from the prediction of the Walton and Chalmers model. First, the misaligned dendrites were able to overgrow the best aligned dendrites. Second, the misaligned grain was able to overgrow the best aligned grain by blocking the best aligned dendrites at the GB. These results on BC samples are well in accordance with the results as shown in Figure 1.

In a BC sample, one grain can be employed to reflect the seed crystal and the other can be employed to reflect a stray grain. From this method we can understand which grain 'wins' in the process of competitive grain growth and more readily predict stray grain defects in SC components. Since such predictions can optimize process design and reduce scrap, casting conditions encompassing those used in an industrial process were adopted in the $\mathrm{BC}$ casting process of the present work.

\section{Experiments}

\section{Materials and Arrangement of Seeds}

The compositions of the materials used in the present work are listed in Table I.

SC seeds from superalloy CMSX-4 were used to initiate the structure development in the casting process. To keep both $<001>$ directions in the $\mathrm{BC}$ samples on the same plane, the $\mathrm{SC}$ seeds were cut from the same SC plate. Each seed was cut into two halves along the sample axis. Subsequently, the seeds were arranged in such a way that the samples with diverging or converging dendrites were produced. Seed A was used to reflect a stray grain and its $<001>$ direction was varied in the experiments. Seed B was used to reflect the seed crystal and its $<001>$ direction 
was constant $\left(15-17^{\circ}\right.$ misaligned from the sample axis). $\theta_{A}$ and $\theta_{B}$ were used to describe misorientation of the $<001>$ direction from the sample axis in seed A and B, respectively. In the present work, seed $\mathrm{A}$ and seed $\mathrm{B}$ were placed on the left and right, respectively. Thus, $\theta_{A}$ and $\theta_{B}$ are defined to be plus when they are inclined from the left to the right. In contrast, $\theta_{A}$ and $\theta_{B}$ are defined to be minus when they are inclined from the right to the left. The details of $\theta_{A}$ and $\theta_{B}$ in the experiments are listed in Table II. It is clear from Table II that $\left|\theta_{A}\right| \leq\left|\theta_{B}\right|$, i.e. grain $\mathrm{A}$ is better aligned to the thermal gradient than grain $\mathrm{B}$.

\section{Casting Experiments}

Casting experiments were carried out using the conventional Bridgman high rate solidification (HRS) technique in a vacuum environment. The SC seeds of $20-25 \mathrm{~mm}$ length were placed in the bottom of a ceramic mould to control the orientations of casting samples. The mould was preheated to $1540{ }^{\circ} \mathrm{C}$, partially melting the seed in a longitudinal thermal gradient. Following a soaking period of 20 minutes, the melt was cast into the preheated mould and the assembly withdrawn at a rate of $3.8 \mathrm{~mm} / \mathrm{min}$ after 3 minutes delay. The cast samples were almost cylindrical with one flat side and had a diameter of $12 \mathrm{~mm}$.

\section{$\underline{\text { Sample Examination }}$}

Macroetching was employed to determine the positions of the grain overgrowth and the melt-back interface. The misorientation of the GB plane from the sample axis $\left(\theta_{G B}\right)$ was determined by $\tan \theta_{G B}=R / L$, where $R$ is the sample radius and $L$ is the distance from the melt-back interface to the position of grain overgrowth. Following the convention used to describe the seed orientations, $\theta_{G B}$ is defined to be plus when the GB plane is inclined from the left to the right, i.e. the GB plane is inclined in such a way that the better aligned grain $\mathrm{A}$ overgrows the misaligned grain $\mathrm{B}$; and $\theta_{G B}$ is defined to be minus when the GB plane is inclined from the right to the left, i.e. the GB plane is inclined in such a way that the misaligned grain B overgrows the better aligned grain A. Subsequently, the casting samples were cut along the sample axis to show the microstructures. $\theta_{A}, \theta_{B}$, primary dendrite arm spacing $\left(\lambda_{1}\right)$, and frequency $(f)$ of dendrite development at the GB were examined by optical metallography on etched samples. $\lambda_{1}$ was determined on the longitudinal plane and on the direction perpendicular to the [001] direction in a grain.

Furthermore, samples of Exp. 6 and 7 were cut perpendicular to the solidification direction and grain structures at different solidified lengths were studied by means of optical microscope or electron back scattered diffraction (EBSD) analysis in a FEI Sirion field emission gun scanning electron microscope, equipped with Oxford EBSD equipment. In the process of EBSD analysis, the sample surface was scanned using an incremental step size of $50 \mu \mathrm{m}$ which is much less than $\lambda_{1}$.

Table I. Nominal compositions of alloys as used in the experiments (in weight percent).

\begin{tabular}{|c|c|c|c|c|c|c|c|c|c|}
\hline $\mathrm{Cr}$ & $\mathrm{Co}$ & $\mathrm{Mo}$ & $\mathrm{W}$ & $\mathrm{Al}$ & $\mathrm{Ti}$ & $\mathrm{Ta}$ & $\mathrm{Re}$ & $\mathrm{Hf}$ & $\mathrm{Ni}$ \\
\hline 6.5 & 9.0 & 0.6 & 6.0 & 5.6 & 1.0 & 6.5 & 3.0 & 0.1 & Bal. \\
\hline
\end{tabular}

Table II. Structural characteristics of the BC samples.

\begin{tabular}{|c|c|c|c|}
\hline Exp. & Seed disposition & $\theta_{A}$ & $\theta_{B}$ \\
\hline Exp.1 & Divergence & $-15^{\circ}$ & $15^{\circ}$ \\
\hline Exp.2 & Divergence & $-5^{\circ}$ & $15^{\circ}$ \\
\hline Exp.3 & Divergence & $0^{\circ}$ & $15^{\circ}$ \\
\hline Exp.4 & Divergence & $8^{\circ}$ & $15^{\circ}$ \\
\hline Exp.5 & Divergence & $15^{\circ}$ & $17^{\circ}$ \\
\hline Exp.6 & Convergence & $9^{\circ}$ & $-16^{\circ}$ \\
\hline Exp.7 & Convergence & $0^{\circ}$ & $-15^{\circ}$ \\
\hline Exp.8 & Convergence & $-9^{\circ}$ & $-16^{\circ}$ \\
\hline
\end{tabular}

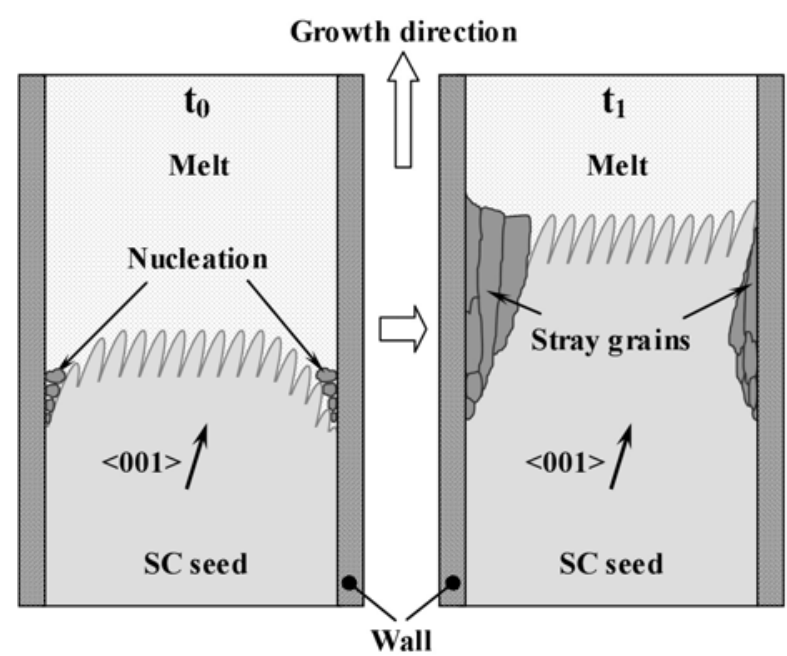

Figure 1. Schematic diagram showing the structure evolution in the SC casting initiated by the SC seed in $[4,5]$.

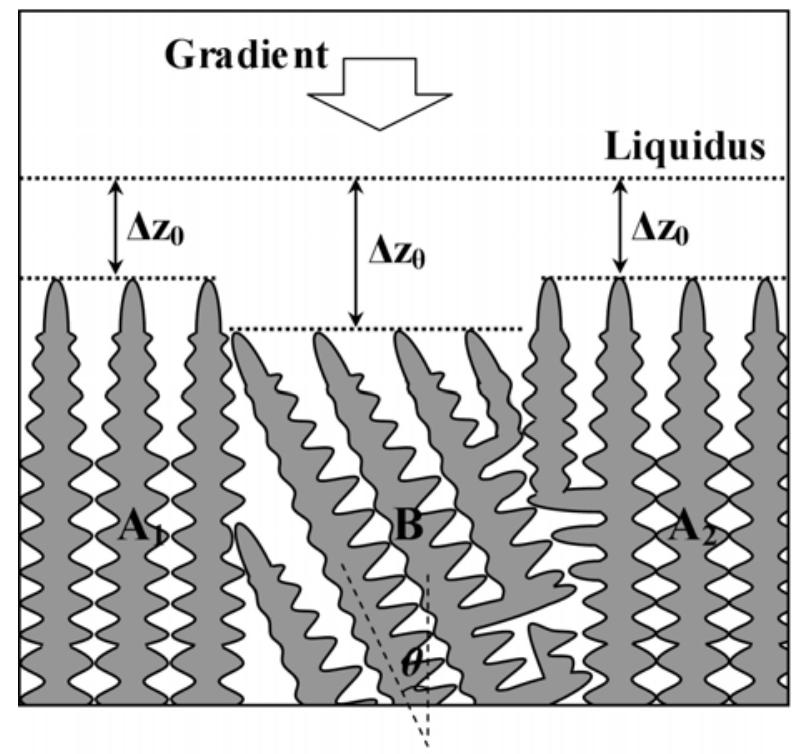

Figure 2. Schematic illustration of the Walton and Chalmers model for competitive grain growth [7]. 


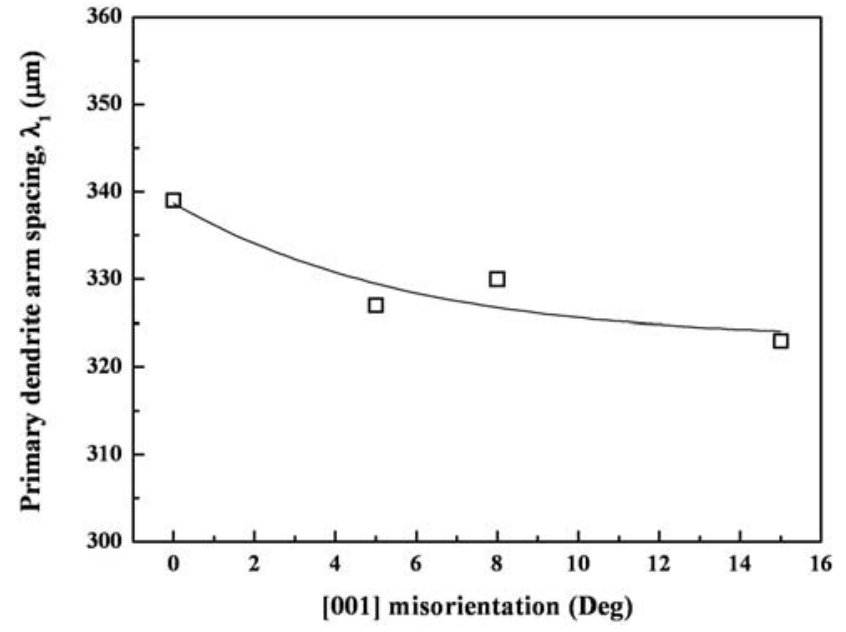

Figure 3. Dependence of $\lambda_{1}$ on [001] misorientation.

\section{Results}

Figure 3 summaries the dependence of $\lambda_{1}$ on [001] misorientation. $\lambda_{1}$ decreased slightly with increasing [001] misorientation.

\section{$\underline{\text { BC Samples with Diverging Dendrites }}$}

Figure 4 (a) shows the optical metallographic microstructure of a BC sample with diverging dendrites. New dendrites developed from grain A and B at the GB and the gap between the diverging grains was filled by these new dendrites. Depending on the development of new dendrites from grain A, the GB was inclined in such a way that grain A overgrew grain B.

The dependence of $\theta_{G B}$ on $\theta_{A}$ in the case of diverging dendrites is summarized in Figure 4 (b). In Exp. 1, $\left|\theta_{A}\right|=\left|\theta_{B}\right|$ gives $\theta_{G B}=0$, i.e. the GB was parallel to the sample axis and there was no grain overgrowth in this experiment. In Exp. 2-5, $\theta_{\mathrm{GB}}>0$ indicates grain
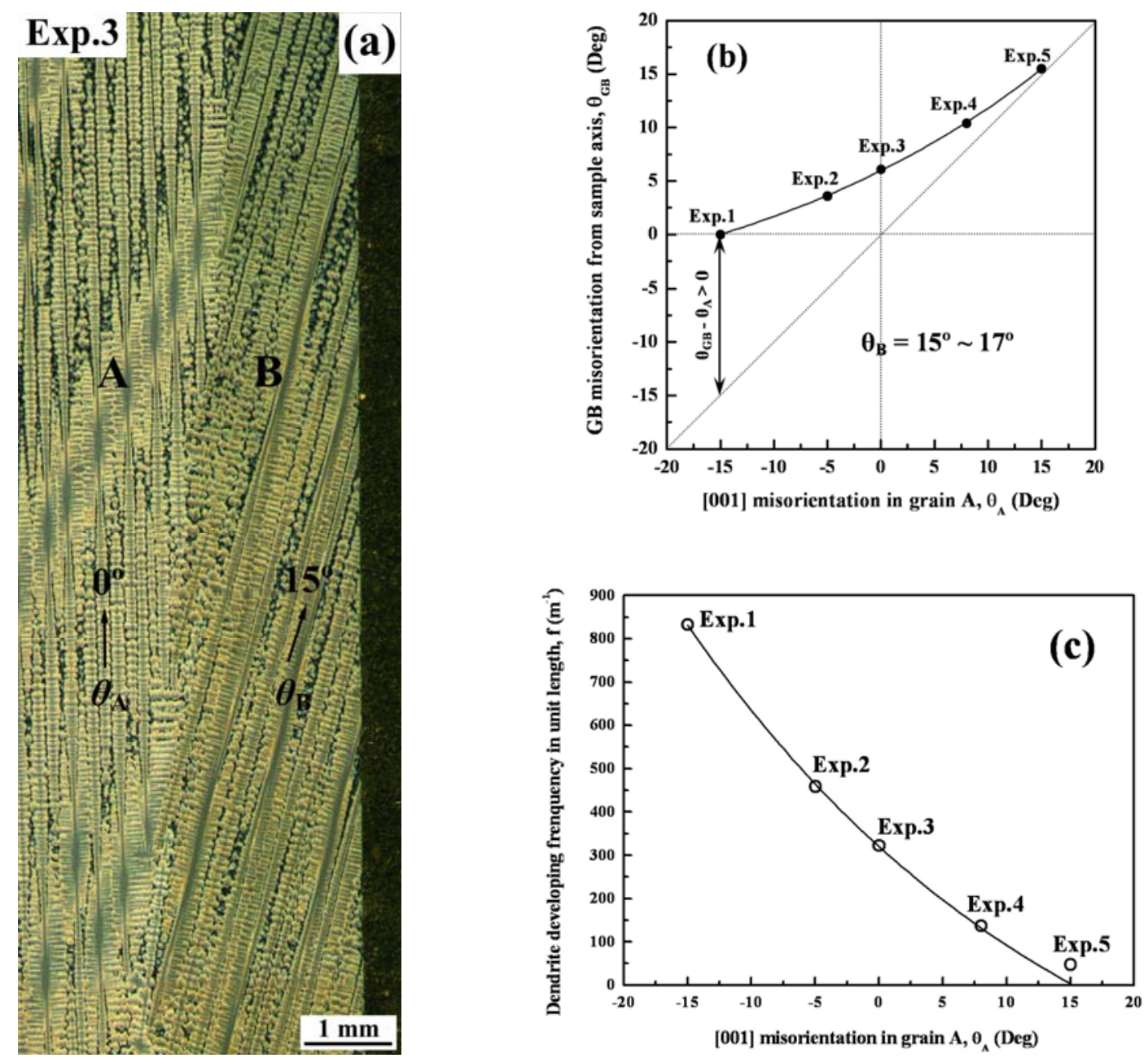

Figure 4. (a) Optical micrograph showing the microstructure in a BC sample with diverging dendrites, (b) dependence of $\theta_{G B}$ on $\theta_{A}$ in the $\mathrm{BC}$ samples with diverging dendrites, and (c) dependence of $f$ on $\theta_{A}$ in the $\mathrm{BC}$ samples with diverging dendrites. 

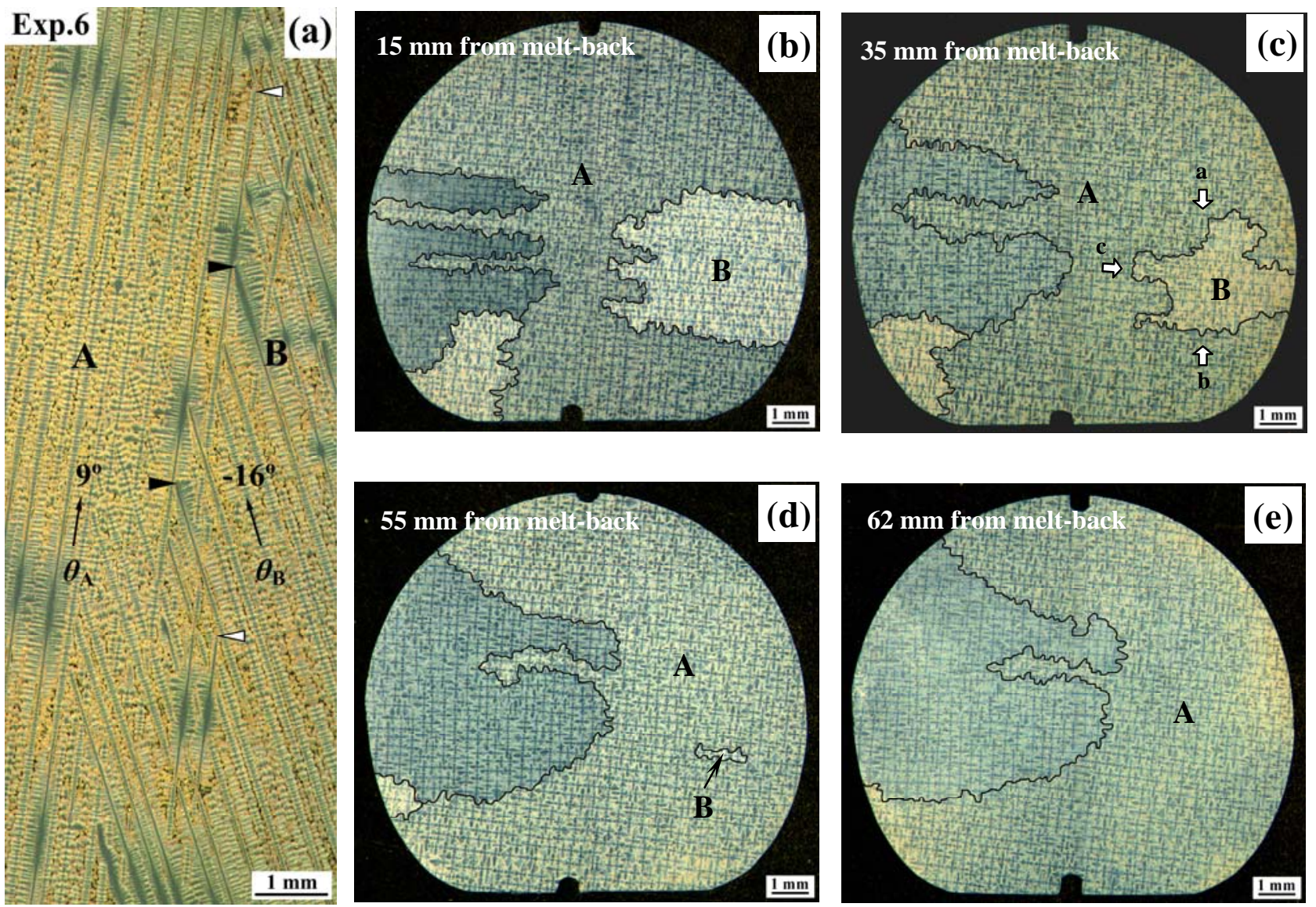

Figure 5. (a) Optical micrograph showing the microstructure on longitudinal section in a BC sample in Exp. 6 with converging dendrites; (b), (c), (d) and (e) optical micrographs showing the microstructures on different sections cut perpendicular to the solidification direction.

A overgrows grain B. In Exp. 1-5, there is the same result of $\theta_{\mathrm{GB}}-$ $\theta_{\mathrm{A}}>0$, which means new dendrites can develop from grain $\mathrm{A}$ at the boundary. As $\theta_{\mathrm{A}}$ became increasingly positive, fewer dendrites developed from grain $\mathrm{A}$. $\theta_{\mathrm{GB}}-\theta_{\mathrm{A}}$ thus became smaller with increasing $\theta_{\mathrm{A}}$. When $\theta_{\mathrm{A}}$ increased to be nearly the same as $\theta_{\mathrm{B}}$, very few dendrites developed from grain $\mathrm{A}$ at the boundary. Consequently, $\theta_{G B}$ was nearly equal to $\theta_{A}$ in Exp. 5 .

Primary dendrite numbers $(f)$ developed from grain $\mathrm{A}$ as the boundary per unit length along the [001] direction in grain A ( $f$ is a ratio of unit length and $l_{001}$ ) is plotted as a function of $\theta_{A}$ in Figure 4 (c). $f$ is reduced with increasing $\theta_{A}$. In Exp. $5 f \neq 0$ is due to $\theta_{\mathrm{B}}=17^{\circ}$ which is a little greater than $\theta_{\mathrm{A}}\left(\theta_{\mathrm{A}}=15^{\circ}\right)$.

\section{BC Samples with Converging Dendrites}

Figure 5 shows the structure evolution in Exp. 6. The microstructure on the sample polished longitudinally (Figure 5(a)) is different from the prediction of the Walton and Chalmers model in two ways. First, not only were the better aligned dendrites able to block the misaligned dendrites (black arrows), but also the misaligned dendrites were able to block the better aligned dendrites (white arrows). Second, new dendrites developed from both grains A and B at the boundary. The positions where new dendrites began appearing was not ahead of the blocked dendrite tips but behind them. Some dendrites developed from the better aligned grain A blocked the misaligned dendrites at the boundary, while dendrites developed from the misaligned grain B cannot generally block the better dendrites. The dendrites in the metallographic samples cut perpendicular to the solidification direction appear as grey crosses in Figure 5 (b)-(e). Grains can be recognized from the different contrast and the gain boundaries are marked by black curves. It is apparent from the microstructure that grain B was overgrown by grain A. However, the grain overgrowth process was not due to the GB displacement on the direction $\mathrm{c}$ but due to the GB displacement on the directions a and $\mathrm{b}$ as marked in Figure 5 (c).

Structure evolution in Exp. 7 is shown in Figure 6. As observed in Exp. 6, not only the better aligned dendrites were able to block the misaligned dendrites, but also the misaligned dendrites were able to block the better aligned dendrites at the boundary, examples of which are marked by short white arrows on the longitudinal polished sample (Figure 6 (a)). Blocking of misaligned dendrites by the better aligned dendrites cannot lead to the GB inclination. However, blocking of better aligned dendrites by the misaligned dendrites can lead to the GB being inclined in such a way that the misaligned grain B overgrew the better aligned grain A. As 

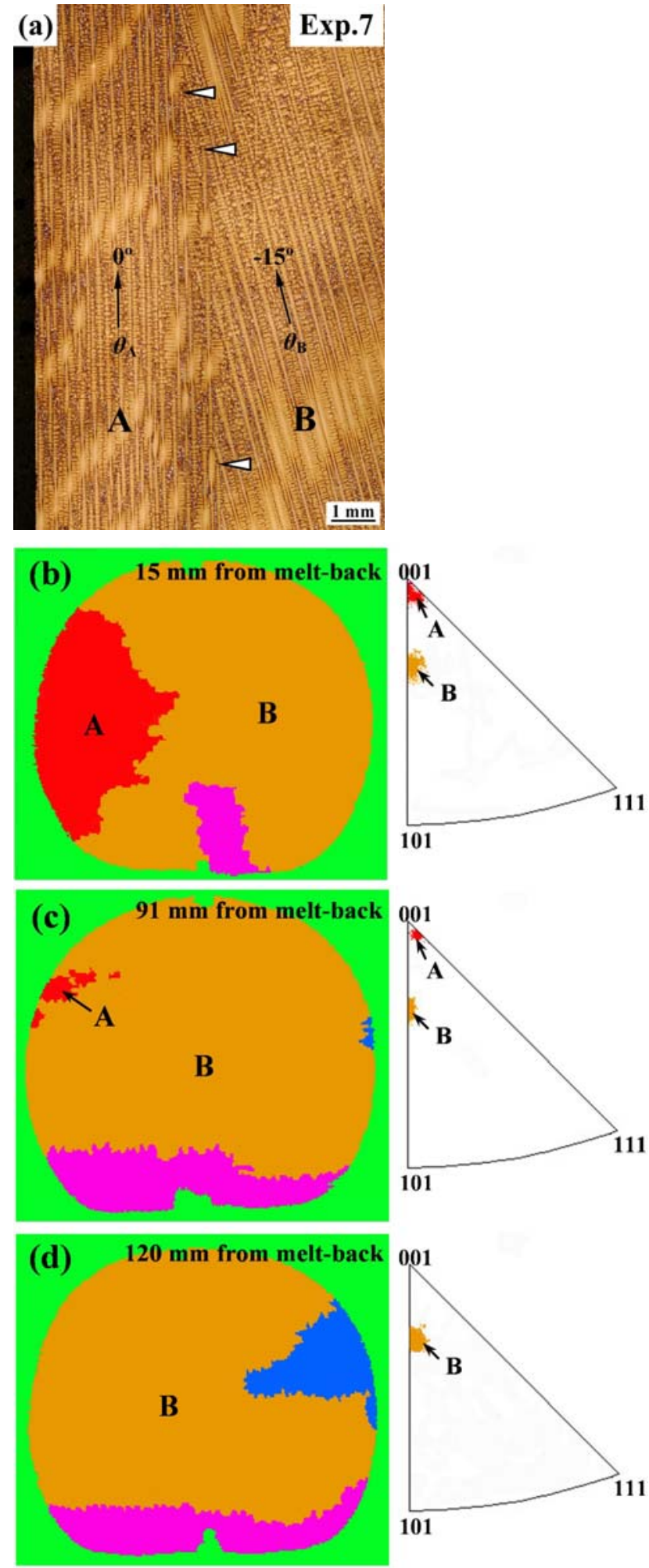

Figure 6. (a) Optical micrograph showing the microstructure on the longitudinal section in a BC sample in Exp. 7 with converging dendrites; (b), (c) and (d) grain maps and corresponding inverse pole figures on different sections cut perpendicular to the solidification direction by means of EBSD analysis.

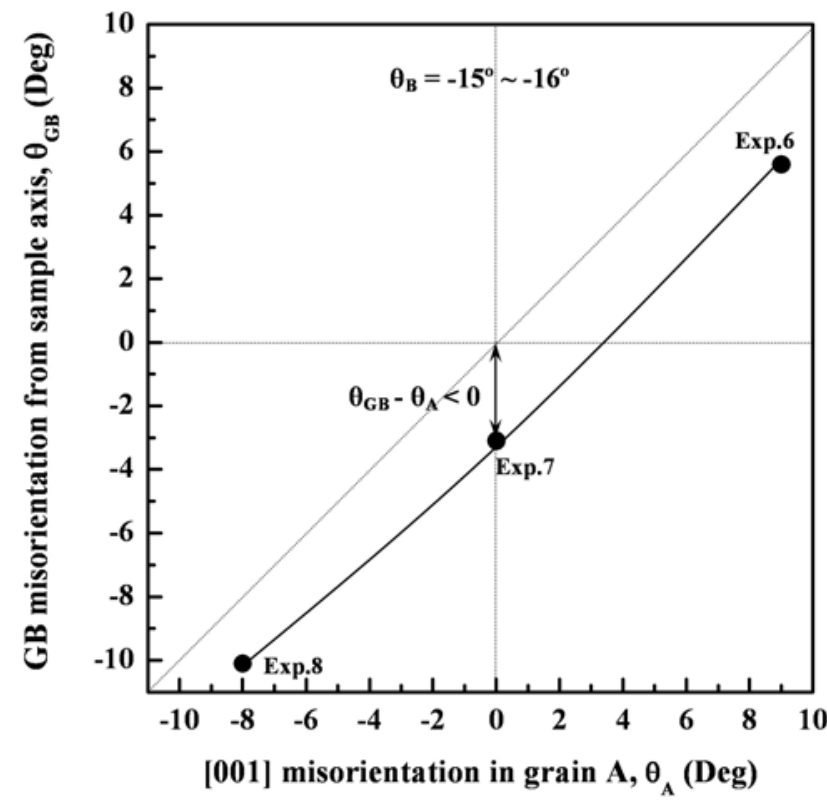

Figure 7. Dependence of $\theta_{G B}$ on $\theta_{A}$ in the $\mathrm{BC}$ samples with converging dendrites.

observed in Exp. 6, new dendrites were able to develop at the GB and the positions where they began appearing was behind the blocked dendrite tips. However, the dendrite number developed at the GB in Exp. 7 was much less than that in Exp. 6. Grain B thus overgrew grain A as evidenced by EBSD analysis (Figure 6 (b), (c) and (d)).

The dependence of $\theta_{G B}$ on $\theta_{A}$ in the case of converging dendrites is summarized in Figure 7. In Exp. 6, $\theta_{\mathrm{GB}}>0$ indicates that the better aligned grain A overgrew the misaligned grain B. In Exp. 7 and $8, \theta_{\mathrm{GB}}<0$ indicates that the misaligned grain $\mathrm{B}$ overgrew the better aligned grain A. In Exp. 6-8, there was the same result of $\theta_{\mathrm{GB}}<\theta_{\mathrm{A}}$, which is different from the suggestion of $\theta_{\mathrm{GB}}=\theta_{\mathrm{A}}$ in the Walton and Chalmers model.

\section{Discussion}

\section{Overgrowth of Diverging Grains}

The results in the samples with diverging dendrites are in accordance to the Walton and Chalmers model. Figure 8 schematically illustrates the dependence of $\theta_{\mathrm{GB}}$ on $\theta_{\mathrm{A}}$ and dendrite development at the GB. The grey bars in the figure represent primary dendrites in grain A. It is clear from Figure 8 that dendrite development from grain A always leads to a GB that is inclined from grain A to grain B (i.e. from the left to the right). Thus $\theta^{\prime}$ is always positive as our definition and $\theta^{\prime}=\tan ^{-1}\left(\lambda_{1} / l_{001}\right)$ $=\tan ^{-1}\left(\lambda_{1} f\right) . \theta_{\mathrm{GB}}, \theta_{\mathrm{A}}$ and $\theta^{\prime}$ follows the equation: $\theta_{\mathrm{GB}}=\theta_{\mathrm{A}}+\theta^{\prime}=$ $\theta_{\mathrm{A}}+\tan ^{-1}\left(\lambda_{1} f\right)$.

The present study indicates, as shown in Figure 1, that any stray grain that is better aligned to the thermal gradient than the seed crystal can survive during competitive growth process with the seed crystal at the diverging side. The stray grains which expand fastest on the transverse section are not the ones whose [001] 
misorientation is well aligned to the thermal gradient, but the ones whose [001] misorientations are closest to the [001] orientation of the seed crystal. The stray grains compete with each other for growth in the casting process. Thus the fastest expanding stray grain may not survive during competitive growth with other stray grains. However, to eliminate all stray grains in a SC component, the fastest expanding stray grain cannot be neglected in the process design.

\section{Overgrowth of Converging Grains and Dendrites}

In the previous work [8], we have reported that the thermal gradient of the casting system is not the reason why the misaligned dendrites are able to overgrow the better aligned dendrites, i.e. the overgrowth process is not due to the inclination of the thermal gradient with respect to the withdrawal direction. The mechanism by which the misaligned dendrites overgrow the better aligned dendrites cannot be stated conclusively from the experimental data. However, solutal interaction of the converging dendrite tips is the most probable factor, i.e. growth of the converging tips at the GB will be retarded due to solutal interactions when they get within the interaction distance. Thus the immediate neighbor of the misaligned dendrite has a chance to overgrow the better aligned dendrite at the GB. In the previous work [8], it was found the dendrites at the GB are behind their immediate neighbors. This result served as evidence of solutal interactions.

In the Walton and Chalmers model, because the misaligned dendrites are not able to overgrow the better aligned dendrites and there is no dendrite development at the GB of converging grains, the GB lies parallel to the better aligned dendrites (i.e. $\theta_{\mathrm{GB}}=\theta_{\mathrm{A}}$ ). Here, we found in the case of converging dendrites the misaligned dendrites are able to overgrow the better aligned dendrites and

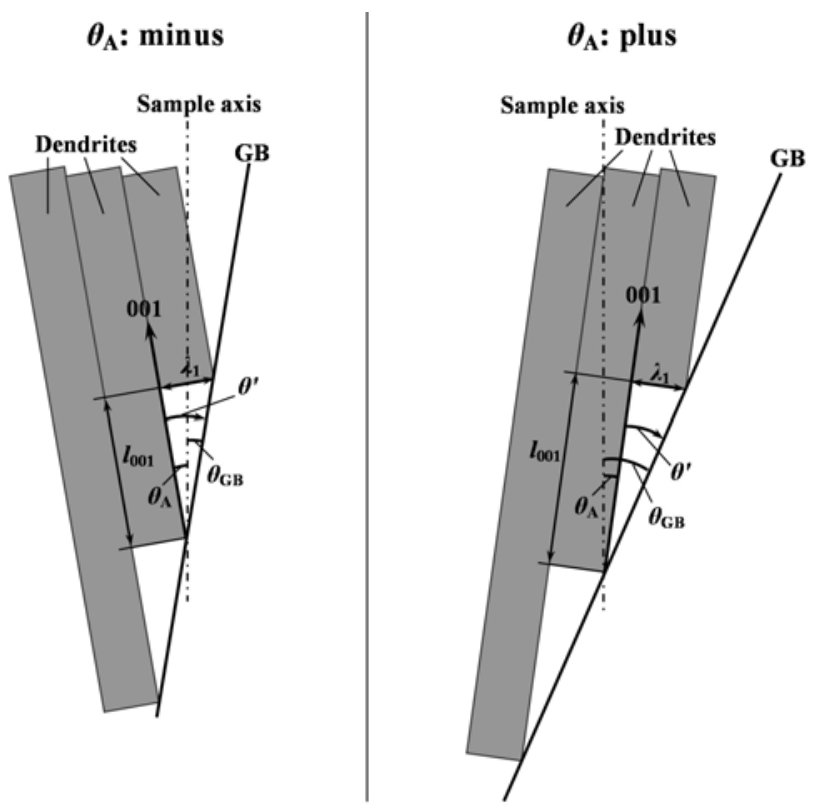

Figure 8. Schematic diagram showing dependence of $\theta_{\mathrm{GB}}$ on $\theta_{\mathrm{A}}$ and dendrite development at the GB. new dendrites are able to develop at the GB. Consequently, the GB does not lie parallel to the better aligned dendrites (i.e. $\theta_{\mathrm{GB}} \neq$ $\left.\theta_{\mathrm{A}}\right)$. As pointed out above, dendrite development from grain $\mathrm{A}$ leads to inclination of the GB by an amount $\theta^{\prime}$ from grain A to grain $\mathrm{B}$ ( $\theta^{\prime}$ is plus). However, blocking of the better aligned dendrites by the misaligned dendrites at the GB leads to the GB is inclined $\theta^{\prime \prime}$ from grain $\mathrm{B}$ to grain $\mathrm{A}$. Following our convention, $\theta^{\prime \prime}$ is minus. Obviously, in the case of converging dendrites $\theta_{\mathrm{GB}}$ can be written as: $\theta_{\mathrm{GB}}=\theta_{\mathrm{A}}+\theta^{\prime}+\theta^{\prime \prime}$. As shown in Figure $7 \theta_{\mathrm{GB}}$ is always less than $\theta_{\mathrm{A}}$ in the case of converging dendrites. This indicates $\theta^{\prime}<\left|\theta^{\prime \prime}\right|$, i.e. the effect of dendrite blocking is greater than the effect dendrite development.

In the case of converging grains, if dendrites at the GB can develop new dendrites on the same longitudinal plane towards the GB by means of branching effect, the positions where the new dendrites begin appearing should be ahead of the blocked dendrite tips in the other grain, but not behind them. Therefore, the microstructures in Figures 5 (a) and 6 (a) show that the new dendrites at the GB of converging grains do not develop from the dendrites on the same longitudinal plane. Figure 9 schematically illustrates the evolution of GB structure in Exp. 6. The white and grey dendrites are on different planes 1 and 2, respectively. Plane 2 is behind plane 1 . When secondary dendrite arms become developed (fine and long) as a result of the high withdrawal speed (such as the withdrawal speed of $3.8 \mathrm{~mm} / \mathrm{min}$ in the present work), the grey dendrites on the plane 2 are able to extend their branches to plane 1 and develop new dendrites on plane 1 as highlighted by arrows $a_{1}$ and $a_{2}$ in Figure 9 (b). Likewise, the white dendrites on the plane 1 are able to extend their branches to plane 2 and develop new dendrites on plane 2 as pointed out by arrows $b_{1}$ and $b_{2}$ in Figure 9 (c). As pointed out above, in Exp. 6 the overgrowth of grain $B$ by grain A mainly depends on the GB displacement along directions $\mathrm{a}$ and $\mathrm{b}$ as marked in Figure 5 (c). Such GB evolution indicates that there is a branching effect from one longitudinal plane to another longitudinal plane in Exp. 6 as illustrated by Figure 9 . Since grain A is better aligned with respect to the gradient, some dendrites developed from grain A can stop the growth of the misaligned dendrites at the GB (for example, dendrite $\mathrm{a}_{1}$ in Figure 9 (b)). However, new dendrites developed from grain B generally cannot stop the growth of the better aligned dendrites at the GB. The dendrite development thus reduces the overgrowth tendency of grain $A$ by grain $B$, and increases the overgrowth tendency of grain $\mathrm{B}$ by grain $\mathrm{A}$.

In Exp. 7 and 8, since the frequency of dendrite impingement between grains $\mathrm{A}$ and $\mathrm{B}$ is less than that in Exp. 6, this merely being due to their geometric disposition to one another, the frequency of branching resolved at longitudinal planes in Exp. 7 and 8 is similarly less than that in Exp. 6. Consequently, far fewer dendrites develop from grains A and B at the GB in Exp. 7 and 8, and the dendrite blocking effect dominated the overgrowth of the better aligned grain A by the misaligned grain $\mathrm{B}$. The geometric dispositions in [8] are similar to that of Exp. 7 and the secondary dendrite arms in [8] were undeveloped due to the use of a slower withdrawal speed $(1 \mathrm{~mm} / \mathrm{min})$. The branching effect between different longitudinal planes was thereby very weak, i.e. dendrites are hard to develop at the GB of converging grains and there is dendrite blocking effect only in [8].

It is clear from the results in the present work that stray grains, as reflected by grains A in Exp. 7 and 8, have no chance to survive during the competitive growth process with the seed crystal B in 


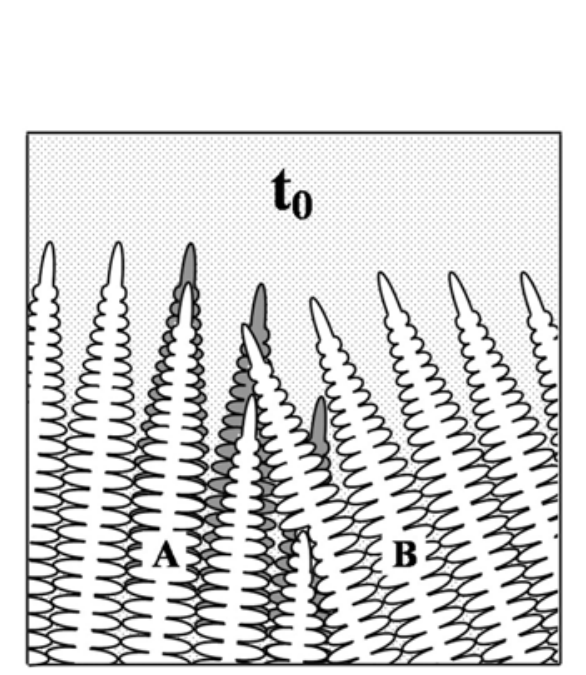

(a)

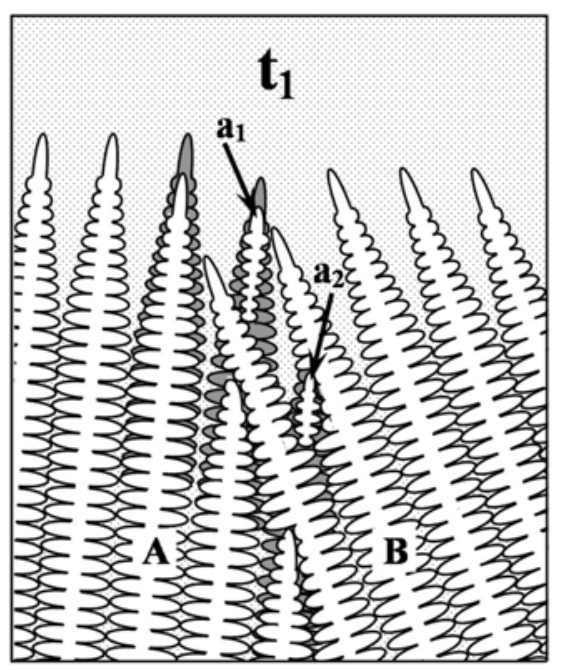

(b)

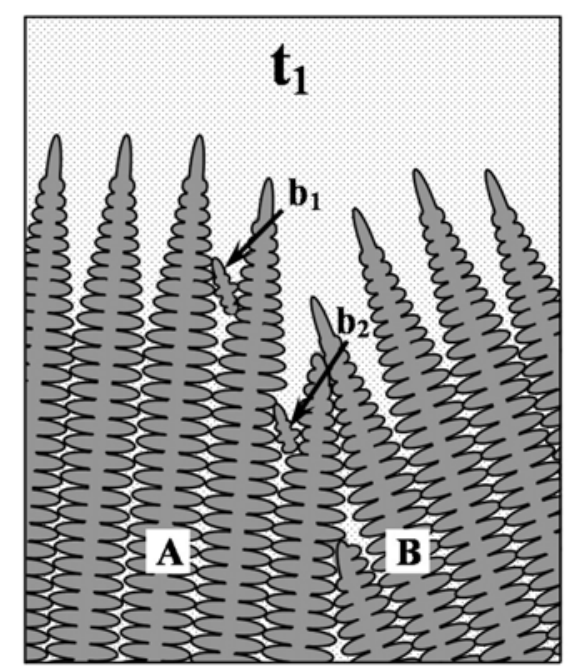

(c)

Figure 9. Schematic diagram showing the structure evolution in Exp. 6. (a) Grain boundary structure at time $\mathrm{t}_{0}$, (b) grain boundary structure at time $t_{1}$, and (c) grain boundary structure on one longitudinal plane at time $t_{1}$.

the practice as shown in Figure 1, converging side. The stray grain as reflected by grain A in Exp. 6 may also have less chance to survive in practice. This would be due to the stray grains being very small in size, and their dendrites are not well developed at the initial state of withdrawal. The above branching effect at different longitudinal planes thus cannot appear on the stray grains. However, the overgrowth effect of stray grains by the seed crystal occurs immediately after withdrawal.

\section{Conclusions}

The overgrowth mechanisms and behaviours of diverging and converging grains in DS castings are different.

In the case of diverging grains, the better aligned grain overgrows the misaligned grain. The grain overgrowth process depends on the development of new dendrites from the better grain at the GB.

In the case of converging grains, the result differs from the prediction of the Walton and Chalmers model. First, when extended secondary arms are developed, new primary and tertiary dendrites are able to develop at the GB depending on the branching effect at different longitudinal planes. This lateral branching effect increases the overgrowth tendency of the misaligned grains by the better aligned grains. Second, on the same longitudinal plane the misaligned dendrites are able to block the better aligned dendrites. The dendrite blocking effect alone can lead to the misaligned grains overgrowing the better aligned grains. Third, grain overgrowth in the case of converging dendrites is controlled by the lateral branching effect and dendrite blocking effect together. Under the casting conditions used in the present work, the dendrite blocking effect is greater than the dendrite developing effect. Consequently, the misaligned grains are able to overgrow the better aligned grains.
The experimental results on $\mathrm{BC}$ samples indicate in seeded $\mathrm{SC}$ casting practice, any stray grain better aligned to the thermal gradient than the seed crystal has a chance to survive on the side where the primary dendrites of the seed diverge from the mould wall. However, stray grains, even those better aligned to the thermal gradient than the seed crystal, have no chance to survive on the side where the primary dendrites of the seed converged to the mould wall.

\section{Acknowledgements}

The authors extend their thanks to Rolls-Royce plc for their financial support of the research which was conducted using facilities within the Casting Partnership with Rolls-Royce. One of the authors (NRG) also thanks EPSRC for their support under grant EP/D505569/1.

\section{References}

1. E.W. Ross, and K.S. O'Hara, "René N4: a first generation single crystal turbine airfoil alloy with improved oxidation resistance, low angle boundary strength and superior long time rupture strength", Superalloys 1996, ed. R.D. Kissinger, D.J. Deye, D.L. Anton, A.D. Cetel, M.V. Nathal, T.M. Pollock, and D.A. Woodford, (Warrendale, PA: The Minerals, Metals \& Materials Society, 1996), 19-26.

2. A.D. Cetel, and D.N. Duhl, "Second-generation nickelbase single crystal superalloy", Superalloys 1988, ed. S. Reichman, D. N. Duhl, G. Maurer, S. Antolovich and C. Lund, (Warrendale, PA: The Minerals, Metals \& Materials Society, 1988), 235-244. 
3. N. Stanford, A. Djakovic, B.A. Shollock, M. McLean, N. D'Souza, and P.A. Jennings, "Seeding of single crystal superalloys - role of seed melt-back on casting defects", Scripta Mater, 50 (2004) 159-163.

4. N. Stanford, A. Djakovic, B.A. Shollock, M. McLean, and N. D'Souza, "Defect grains in the melt-back region of CMSX-4 single crystal seeds", Superalloys 2004, ed. K.A. Green, T.M. Pollock, H. Harada, T.E. Howson, R.C. Reed, J.J. Schirra, and S. Walston, (Warrendale, PA: The Minerals, Metals \& Materials Society, 2004), 719-726.

5. N. D'Souza, P.A. Jennings, X.L. Yang, H.B. Dong, and M. McLean, "Seeding of single-crystal superalloys role of constitutional undercooling and primary dendrite orientation on stray grain nucleation and growth", Metall Mater Trans, 36B (2005), 657-666.

6. D. Walton, and B. Chalmers, "The origin of the preferred orientation in the columnar zone of ingots", Trans Metall Soc AIME, 215 (1959), 447-457.

7. M. Rappaz, C.A. Gandin, J.L. Desbiolles, and P. Thevoz, "Prediction of grain structure in various solidification processes", Metall Mater Trans, 27A (1996), 695-705.

8. Y.Z. Zhou, A. Volek, and N.R. Green, "Mechanism of competitive grain growth in directional solidification of a nickel-base superalloy”, Acta Mater. 2008, In press. 